\title{
Mental illness-related disparities in length of stay: Algorithm choice influences results
}

\author{
Susan M. Frayne, MD, MPH; ${ }^{1-3^{*}}$ Eric Berg, MS $;^{1-2}$ Tyson H. Holmes, PhD ${ }^{4}{ }^{K a a j a l ~ L a u n g a n i, ~ B A ; ~}{ }^{1}$ Dan R. \\ Berlowitz, MD, MPH $;{ }^{5}$ Donald R. Miller, ScD $;^{5}$ Leonard Pogach, MD, MBA; ${ }^{6}$ Valerie W. Jackson, MPH; ${ }^{1-2}$ \\ Rudolf Moos, PhD $\mathbf{P}^{1,3-4}$ \\ ${ }^{1}$ Center for Health Care Evaluation, Department of Veterans Affairs (VA) Palo Alto Health Care System, Palo Alto, CA; \\ ${ }^{2}$ Division of General Internal Medicine, Stanford University, Palo Alto, CA; ${ }^{3}$ Center for Primary Care and Outcomes \\ Research, Stanford University, Palo Alto, CA; ${ }^{4}$ Department of Psychiatry and Behavioral Sciences, Stanford University, \\ Palo Alto, CA; ${ }^{5}$ Center for Health Quality, Outcomes, and Economic Research, Edith Nourse Rogers Memorial Veterans \\ Hospital, Bedford, MA; and Boston University School of Public Health, Boston, MA; ${ }^{6}$ Center for Healthcare Knowledge \\ Management, VA New Jersey Health Care System, East Orange, NJ; and University of Medicine and Dentistry of New \\ Jersey-New Jersey Medical School, Newark, NJ
}

\begin{abstract}
Methodological challenges arise when one uses various Veterans Health Administration (VHA) data sources, each created for distinct purposes, to characterize length of stay (LOS). To illustrate this issue, we examined how algorithm choice affects conclusions about mental health condition (MHC)-related differences in LOS for VHA patients with diabetes nationally $(n=$ 784,321 ). We assembled a record-level database of all fiscal year (FY) 2003 inpatient care. In 10 steps, we sequentially added instances of inpatient care from various VHA sources. We processed databases in three stages, truncating stays at the beginning and end of FY03 and consolidating overlapping stays. For patients with MHCs versus those without MHCs, mean LOS was 17.7 versus 13.6 days, respectively ( $p<0.001$ ), for the crudest algorithm and 37.2 versus 21.7 days, respectively ( $p<0.001$ ), for the most refined algorithm. Researchers can improve the quality of data applied to VHA systems redesign by applying methodological considerations raised by this study to inform LOS algorithm choice.
\end{abstract}

Key words: algorithms, databases, Department of Veterans Affairs, episode of care, healthcare disparities, health services research, human, length of stay, mental disorders, outcome and process assessment, patient discharge, physician's practice patterns, rehabilitation, reproducibility of results, veterans, veterans hospitals.

\section{INTRODUCTION}

Health services researchers often use administrative data for characterizing length of stay (LOS) to address a range of objectives. For example, they may examine how LOS (as a dependent variable) varies as a function of patient characteristics (e.g., age, race, insurance status, presence of comorbidity), processes of care (e.g., speed of emergency department response, types of medications administered or interventions applied, discharge protocols, etc.), or institutional characteristics (e.g., teaching hospital, mental health facility, etc.) [1-7]. Alternatively,

Abbreviations: DEpiC $=$ Diabetes Epidemiology Cohort, DSS = Decision Support System, EXT = extended care, FY = fiscal year, ICD-9 = International Classification of Diseases-9th Revision, LOS = length of stay, MHC = mental health condition, OBS = observation, OPAT = outpatient file, VHA = Veterans Health Administration.

*Address all corr espondence to Susan M. Fr ayne, M D, MPH; Center fo $r$ Health Ca re Eva luation, 795 Willow Road (152-MPD), Palo Alto, CA 940 25; 650-493-5000, ext 23369; fax: 650-617-2690. Email: sfrayne@stanford.edu DOI:10.1682/JRRD.2009.08.0112 
they may examine LOS as a potential explanatory variable for predicting other outcomes [8] or they may restrict their cohort to patients meeting specific LOS criteria [9]. Furthermore, accurate identification of intervals of inpatient care is required for studies using an episodes-of-care approach [10].

The concept of LOS is simple: time from admission to discharge. However, a number of methodological considerations arise when Veterans Health Administration (VHA) data are used for calculating LOS. First, goals of the project must be carefully considered, because this will influence the algorithm selected. Is the focus on acute or long-term care, on medical-surgical or mental health stays? Is the objective to examine total LOS across multiple years or LOS during a particular interval of study? Second, the algorithm must account for technical, data-quality issues. These include duplicate records, overlapping or sequential inpatient stays, transfers between different inpatient units, and inpatient stays that are recorded in a subsequent year.

Despite that numerous studies focus on LOS, these subtleties of LOS calculation have received little attention. This oversight could have serious implications: algorithm choice can influence conclusions in health services studies [11-13], although to our knowledge this possibility has not been studied in the specific case of LOS. As VHA leadership increasingly seeks to obtain accurate estimates of healthcare costs and use evidence to guide strategic planning decisions, it is critical that the evidence base supporting those decisions be as accurate as possible.

One example of a clinical scenario wherein LOS algorithm choice could influence conclusions is mental health condition (MHC)-related differences in inpatient care use. Prior studies both within and outside the VHA have documented that, compared with patients without MHCs, patients with MHCs tend to use more inpatient care [6,1419]. Thus, patients with MHCs represent a particularly high-intensity, high-cost group likely to merit special attention by VHA policy makers. However, some characteristics of the way patients with MHC receive inpatient care may make their VHA records disproportionately susceptible to variation in algorithm choice. For example, patients with MHC might be more likely to experience more complex patterns of inpatient care (e.g., transferring between a medical unit and a psychiatric unit during the course of a single hospitalization episode), or to receive care in extended-care settings, where stays can be long and can span multiple fiscal years (FYs). Such factors could potentially influence LOS calculations differently for patients with MHC versus those without MHC.

We used VHA administrative data to examine how application of incrementally more refined algorithms for calculating LOS during 1 year of care affected conclusions about mean LOS in a national cohort of VHA patients with diabetes. Then, as an illustrative example of the practical implications of such methodological decisions, we examined whether the magnitude of observed mental illness-related disparities in mean LOS varied as a function of LOS algorithm applied.

\section{METHODS}

\section{Study Context}

This work is part of a larger study examining the effect of MHC on processes of outpatient diabetes care in FY03. Because the focus of that study is on outpatient care, we wished to identify (and ultimately exclude from the larger study) patients who were institutionalized (i.e., on inpatient status) for the majority of FY03. Therefore, our goal was to identify, for each patient in our cohort, all days in FY03 during which the patient was on inpatient status (acute care or extended care). We were not seeking to characterize total LOS for the patients in our cohort (which could have spanned multiple years), but only those inpatient days that occurred during FY03. The process of creating our LOS variable and the effect of algorithm choice on conclusions about MHC-related differences in LOS is the focus of the present study.

\section{Subjects}

The cohort was drawn from the FY02 Diabetes Epidemiology Cohort (DEpiC), a census of patients with diabetes in VHA nationally. DEpiC is used extensively for VHA epidemiological and health services research [20]. DEpiC identifies patients with diabetes based on the presence of at least one instance of an antiglycemic prescription or at least two instances of a diabetes International Classification of Diseases-9th Revision (ICD-9) code in inpatient or outpatient records. Among the 911,451 FY02 DEpiC members who were veterans, used VHA outpatient care at least once in FY02, and were alive as of the first day of FY03, we selected the 784,321 whose MHC status could be verified, as described next (in subsidiary analyses, we included the full 911,451 subjects, including those with "MHC Possible" status). 


\section{Steps to Assemble Raw Record-Level Database of Inpatient Stays}

We started by creating a record-level file containing every instance of inpatient care recorded in any inpatient database available in centralized VHA files. We selected only records that contained at least 1 day of inpatient care in FY03. We also deleted duplicate records. In 10 sequential "steps," we pulled all nonduplicate inpatient records containing any FY03 inpatient care for patients in our cohort from the following FY03 files:

Step 1. Bedsection file, which represents acute care hospital stays.

Step 2. OBS (Observation) file, which represents short (e.g., overnight) acute care stays during which the patient is observed regarding the potential need for admission to an acute care bed.

Step 3. EXT (Extended Care) file, which represents long-term care stays (such as rehabilitation stays or nursing home stays).

Step 4. Census file (for Bedsection, OBS, and EXT), which include records for all patients who still held inpatient status on the last day of the FY, and thus for whom a discharge date was not available when the files for that FY were created.

Step 5. Non-VHA file.

Step 6. Fee basis file.

(These latter two files reflect care received outside of VHA but with funding for the care provided by VHA.)

We then searched FY04 and FY05 files for any records that included some FY03 care:

Step 7. Sources 1 through 5, FY04.

Step 8. Fee basis FY04 file (presented separately from other FY04 files to emphasize that fee basis files are more likely to contain "late entry" records from prior years).

Step 9. Sources 1 through 5, FY05.

Step 10. Fee basis FY05 file.

\section{Stages of Processing Record-Level Database of Inpatient Stays}

Next, we processed this raw database in sequential "stages." Stage A represented the raw file at any given step. In stage B, we deleted pre-FY03 and post-FY03 care. Specifically, for records with an admission date earlier than the first day of FY03, we deleted any days preceding FY03 (i.e., we modified the record to begin on the first day of FY03), because we were interested in days of care during FY03, not total LOS for the patient across multiple years.
Similarly, for records with a discharge date later than the last day of FY03, we modified the record to end on the last day of FY03.

In stage C, we addressed overlapping stays. Several types of overlap were observed, as illustrated in Figure 1. In some cases, the entire stay (admission date through discharge date) was contained within the time interval of another record. This might happen, for example, if a patient in a rehabilitation unit was temporarily transferred to an acute care observation bed for an intercurrent illness like pneumonia. If the patient was not formally discharged from the rehabilitation facility prior to the transfer, then the time interval of the short-term stay (appearing in the OBS file) could be bracketed by the interval of the longterm stay (appearing in the EXT file). In other cases an overlap occurred (e.g., the admission date of one record fell between the admission and discharge dates of a subsequent record, or the discharge date of a record fell between the admission and discharge date of a subsequent record). In other cases, contiguous admissions occurred (i.e., the discharge date of one record was the same as the admission date of a subsequent record). For all these overlap cases (which could involve a pair of records or even three or more records), we created a single contiguous episode of FY03 inpatient care by assigning the admission date to be the first admission date in FY03 among the overlapping records and the discharge date to be the last discharge date in FY03 among the overlapping records. The resulting file at step 10, stage $\mathrm{C}$, was our final recordlevel file of inpatient stays.

\section{Variables}

We calculated LOS for each record as the number of days from its start through end dates. At each step/stage, we calculated a cumulative LOS for each patient by adding the record-level LOS for all records included in that step/stage.

To identify patients with MHC, we used the Agency for Health Research and Quality's Clinical Classifications Software (with minor modifications) to generate a list of ICD-9 codes indicating the presence of MHC [21]. A patient was assigned a "Yes" for MHC status if he/she had at least one instance of an MHC ICD-9 code in any inpatient record or outpatient face-to-face clinic visit at baseline (FY01-02) and at least one confirmatory ICD-9 in the study period (FY03). If he/she had no instance of an MHC ICD-9 in FY01 through 03, then he/she was assigned MHC status “No.” Otherwise, MHC status was 
considered "Possible." That is, the MHC Possible group represents those patients who had an MHC diagnosis in the baseline period or in the study period, but not both.

\section{Contiguous records, $n=65,102$}

a

b

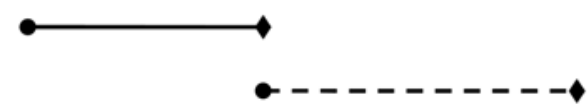

II. Record within another record, $n=11,711$

a

b

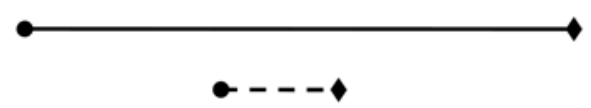

III. Overlapping discharge dates, $n=2,887$

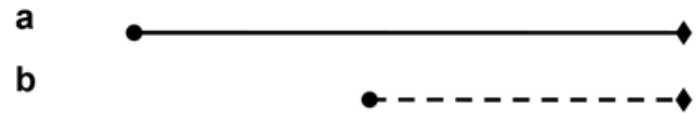

\section{Overlapping admission dates, $n=1,569$}

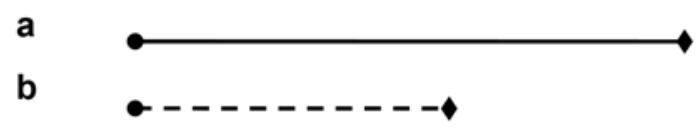

\section{Partial overlap across records, $n=1,575$}

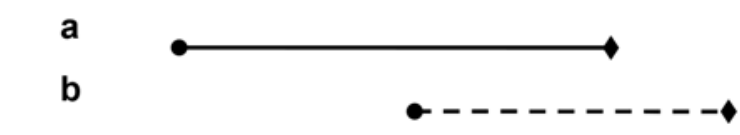

Time

$a=$ Index Record

b = Overlapping Record

- Admission Date

$\rightarrow$ Discharge Date

Figure 1.

Patterns I-V of overlap between pairwise records of an individual patient and record-level frequency of each pattern at step 10, stage C.
Cases with MHC Possible status were excluded from main analyses; this allowed us to compare LOS in two more sharply defined groups (MHC Yes vs MHC No).

\section{Analysis}

We tabulated the number of records and calculated mean LOS within each cell of a $10 \times 3$ matrix representing the steps and stages of database development. Next, in each cell, we calculated mean LOS as a function of MHC status. We then calculated the difference $(\Delta)$ in mean LOS among patients with MHC versus those without MHC and compared mean LOS for the MHC Yes versus MHC No groups using a two-sample $t$-test. We applied Bonferroni correction for compounding of Type I error across multiple comparisons. Results of hypothesis tests are declared statistically significant for $p<0.05$ after Bonferroni correction.

\section{RESULTS}

Among the 784,321 patients with diabetes in the full cohort, 152,591 were identified as having evidence of an MHC diagnosis (MHC Yes). Among the subset of 92,255 patients who received any inpatient care in FY03 (based on step 10, stage C), 39,452 had MHC Yes. Table 1 presents the age, sex, Physical Comorbidity Index score (a count from 0-35, developed for case mix adjustment in VHA patients [22-23]), and primary care use in the full cohort and in the subset who used inpatient care, by MHC status.

Table 2 catalogs the number of records and LOS at each step/stage in the database assembly process. The cumulative number of patients who are identified as having received inpatient care in FY03 (based on stage C) increases progressively from step 1 to step 10 (as do the number of records). For example, when the OBS file was added to the Bedsection file, an additional 10,660 records were added for stays that did not perfectly duplicate a Bedsection file stay for that patient. This is expected, because additional evidence of inpatient care is added at each step. More noteworthy is that some steps contribute more records than others.

The number of records does not change at stage $B$ (compared with stage A), because this processing step truncates records (to include only inpatient days during FY03) but does not delete records. However, at stage C (record consolidation), the number of records drops substantially, because overlapping stays are merged into a single, longer stay. 
Table 1.

Characteristics of cohort by mental health condition (MHC) status (full cohort and subset who used Veterans Health Administration inpatient care).

\begin{tabular}{|c|c|c|c|c|}
\hline \multirow{2}{*}{ Characteristic } & \multicolumn{2}{|c|}{ Full Cohort, $n=784,321$} & \multicolumn{2}{|c|}{ Inpatient Users, ${ }^{*} n=92,255$} \\
\hline & MHC Yes & MHC No & MHC Yes & MHC No \\
\hline $\bar{n}$ & 152,591 & 631,730 & 39,452 & 52,803 \\
\hline Age (years, mean \pm SD) & $62.1 \pm 11.6$ & $69.6 \pm 10.3$ & $61.4 \pm 11.9$ & $69.1 \pm 10.5$ \\
\hline Male (\%) & 96.4 & 98.5 & 96.1 & 98.4 \\
\hline Physical Comorbidity Index (mean \pm SD) & $3.6 \pm 2.4$ & $2.8 \pm 2.0$ & $4.6 \pm 2.8$ & $4.5 \pm 2.7$ \\
\hline Used Primary Care in FY03 (\%) & 93.6 & 86.8 & 90.6 & 91.7 \\
\hline
\end{tabular}

Table 2 .

Effect of sequential data assembly steps and data cleaning stages on number of patients identified as having received inpatient care and on count of inpatient records and mean length of stay (LOS).

\begin{tabular}{|c|c|c|c|c|c|c|c|c|}
\hline \multirow{2}{*}{ Step } & \multirow{2}{*}{$\begin{array}{c}\text { Patients }^{*} \\
\text { (n) }\end{array}$} & \multirow{2}{*}{$\begin{array}{c}\text { Records } \\
\text { Added in } \\
\text { Step }(n)\end{array}$} & \multicolumn{3}{|c|}{ Number of Records } & \multicolumn{3}{|c|}{ LOS (days), Mean \pm SD } \\
\hline & & & Stage A & Stage B & Stage $C$ & Stage A & Stage $B$ & Stage C \\
\hline 1. Bedsection FY03 & 77,817 & 173,707 & 173,690 & 173,690 & 127,566 & $15.3 \pm 21.1$ & $14.8 \pm 20.1$ & $14.2 \pm 19.4$ \\
\hline 3. EXT FY03 & 85,198 & 14,844 & 199,194 & 199,194 & 140,283 & $27.3 \pm 103.8$ & $21.7 \pm 37.8$ & $20.7 \pm 36.1$ \\
\hline 4. Census FY03 & 86,990 & 6,990 & 206,184 & 206,184 & 143,193 & $37.6 \pm 198.4$ & $26.7 \pm 53.1$ & $25.6 \pm 51.2$ \\
\hline 5. Non-VHA FY03 & 89,135 & 5,438 & 211,622 & 211,622 & 146,723 & $37.2 \pm 196.2$ & $26.5 \pm 52.8$ & $25.4 \pm 50.9$ \\
\hline 7. FY04 Records & 90,689 & 5,898 & 232,627 & 232,627 & 148,905 & $58.3 \pm 370.4$ & $35.7 \pm 91.2$ & $28.0 \pm 57.4$ \\
\hline 8. Fee FY04 & 92,068 & 6,829 & 239,456 & 239,456 & 151,891 & $58.4 \pm 368.5$ & $36.1 \pm 93.0$ & $28.3 \pm 58.9$ \\
\hline 9. FY05 Records & 92,181 & 1,569 & 241,025 & 241,025 & 151,980 & $74.9 \pm 518.8$ & $39.4 \pm 113.4$ & $28.4 \pm 59.3$ \\
\hline 10. Fee FY05 & 92,255 & 293 & 241,318 & 241,318 & 152,157 & $74.8 \pm 518.6$ & $39.4 \pm 113.4$ & $28.4 \pm 59.3$ \\
\hline
\end{tabular}

Note: To create table, we started with step 1 and completed cells across each stage sequentially. Then, for the step 2 analyses, we started with records from steps 1 and 2 and completed cells across each stage sequentially. Analyses for each subsequent step similarly included records from all prior steps. Stages were stage A (original record), stage B (delete days prior to first day of FY03 and after last day of FY03), and stage C (consolidate overlapping stays).

${ }^{*}$ Reflects cumulative number of patients who received inpatient care in FY03 at each step at stage C. Inpatient records were drawn from patients in analytical cohort $(n=784,321)$.

EXT = extended care, $\mathrm{FY}=$ fiscal year, $\mathrm{OBS}=$ observation, $\mathrm{SD}=$ standard deviation, $\mathrm{VHA}=$ Veterans Health Administration.

Consistent with these observations, mean LOS at stage $\mathrm{C}$ increased progressively with sequential steps (i.e., as more sources of data were added), except at step 2 (where patients with short OBS stays were added) and at step 5 (where patients with non-VHA stays were added). Similarly, mean LOS decreased progressively with sequential stages. That is, mean LOS decreased from stage A to stage B as non-FY03 days were deleted (which would be relevant to a study like ours that focuses on care received in a single FY). Mean LOS also decreased from stage $\mathrm{B}$ to stage $\mathrm{C}$ as overlapping days were deleted (which would be relevant to the accuracy of the LOS estimate in any study design). Across the $10 \times 3$ matrix, mean LOS ranged from 13.8 to 74.9 days.
Table 3 presents LOS by MHC status at every step/ stage in the database assembly process. The calculated difference $(\Delta)$ in mean LOS between the MHC Yes and the MHC No groups varied markedly by algorithm and was statistically significant $(p<0.001)$ at every step/stage. Correction for multiple comparisons did not statistically affect any findings significantly. As illustrated in Figure 2, step $1, \Delta=4.1$ at stage A and 3.8 at stage $\mathrm{C}$. In contrast, at step $10, \Delta=57.8$ at stage A and 15.5 at stage $\mathrm{C}(p<0.01$ for both between-algorithm comparisons of the values of $\Delta$ ).

To obtain the LOS in stage $C$, for each pair of overlapping records, we generated a single record by setting the FY03 admission date as the earliest of the two admission dates and the FY03 discharge date as the latest of the two 
Table 3.

Effect of sequential data assembly steps/data cleaning stages on fiscal year (FY) 2003 length of stay (LOS) calculations by mental health condition (MHC) status.

\begin{tabular}{|c|c|c|c|c|c|c|c|c|c|}
\hline \multirow{2}{*}{ Step } & \multicolumn{3}{|c|}{ Stage A } & \multicolumn{3}{|c|}{ Stage B } & \multicolumn{3}{|c|}{ Stage C } \\
\hline & MHC Yes & MHC No & $\Delta$ & MHC Yes & MHC No & $\Delta$ & MHC Yes & MHC No & $\Delta$ \\
\hline 1. Bedsection FY03 & 17.7 & 13.6 & 4.1 & 17.0 & 13.2 & 3.8 & 16.4 & 12.6 & 3.8 \\
\hline 3. EXT FY03 & 36.6 & 20.4 & 16.2 & 27.5 & 17.4 & 10.1 & 26.4 & 16.5 & 9.9 \\
\hline 4. Census FY03 & 51.6 & 27.2 & 24.4 & 34.0 & 21.3 & 12.7 & 32.7 & 20.3 & 12.4 \\
\hline 7. FY04 Records & 82.1 & 40.5 & 41.6 & 47.1 & 27.3 & 19.8 & 36.7 & 21.5 & 15.2 \\
\hline 8. Fee FY04 & 82.3 & 40.5 & 41.8 & 47.8 & 27.4 & 20.4 & 37.1 & 21.6 & 15.5 \\
\hline 9. FY05 Records & 108.0 & 50.2 & 57.8 & 52.5 & 29.6 & 22.9 & 37.3 & 21.7 & 15.6 \\
\hline 10. Fee FY05 & 107.9 & 50.1 & 57.8 & 52.4 & 29.6 & 22.8 & 37.2 & 21.7 & 15.5 \\
\hline
\end{tabular}

$\overline{\text { Note: Every difference }(\Delta) \text { between mean LOS for MHC Yes vs MHC No in this table is statistically significant at } p<0.001 \text {. Stages were stage A (original record), }}$ stage B (delete days prior to first day of FY03 and after last day of FY03), and stage C (consolidate overlapping stays). Two sample $t$-tests were conducted for two key comparisons in this table: comparing within step 1 for stage A vs stage $C$ and within step 10 for stage A vs stage $C$ ( $p<0.01$ for both comparisons). $\Delta=$ mean LOS (MHC Yes) minus mean LOS (MHC No), EXT = extended care, OBS = observation, VHA = Veterans Health Administration.

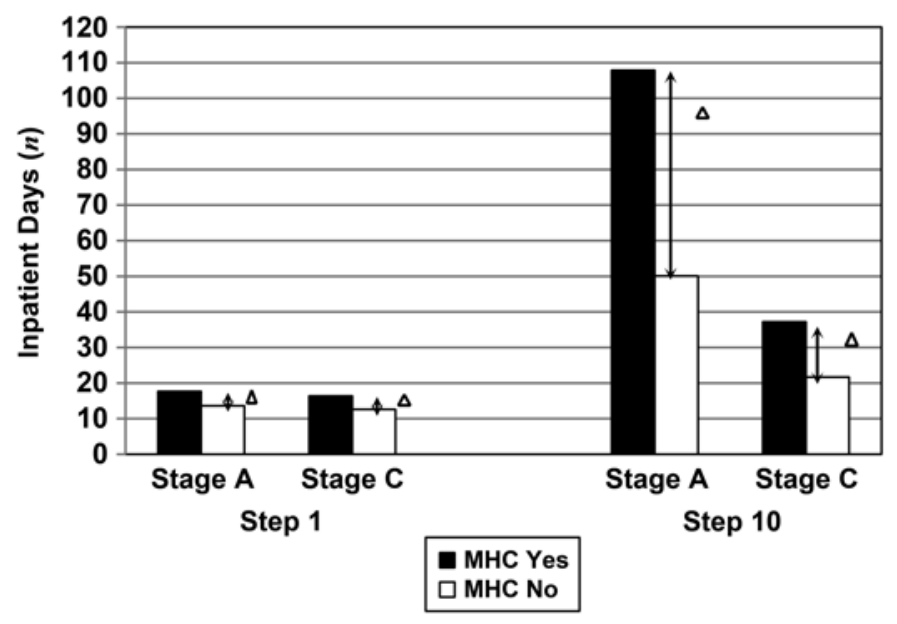

Figure 2.

Effect of sequential data assembly steps and data cleaning stages on fiscal year 2003 number of inpatient days. MHC = mental health condition, $\Delta=$ mean length of stay (LOS) (MHC Yes) - mean LOS (MHC No).

discharge dates. We repeated this process iteratively until all pairwise overlaps were addressed. This data processing stage was the most involved, because it needed to account for multiple potential overlap patterns, as illustrated schematically in Figure 1. The most common overlap pattern (pattern I) was contiguous records, i.e., where the discharge date of one record was the admission date of the following record. This pattern would happen, for example, if a patient were admitted to one bed section (e.g., to the Psychiatry Department for suicidal ideation) and then transferred to another bed section (e.g., to General Medicine for a hospital-acquired infection). Of note, we used the Bedsection files for these analyses. VA Bedsection files create a new record each time a patient transfers to a different clinical service ("bedsection”) during a hospital stay. This is in contrast to the VA Main files, which create a new record for each stay; all contiguous bedsection stays are combined in a single record. Had we used the Main file instead of the Bedsection file, we expect that we would not have encountered this particular form of overlap. Other overlap patterns were also observed, as Figure 1 shows. Of note, step 10, stage B, yielded LOSs of more than 365 days for 3.2 percent of the MHC Yes group and 1.4 percent of the MHC No group, clearly representing a residual problem with the algorithm; in contrast, no patient had LOS greater than 365 days at stage $C$. This finding supports the importance of the stage $C$ processing.

In a subsidiary analysis, we found that both the admission and discharge dates fell within FY03 for 91 percent of records at step 10 , stage $A$. In those instances, the full LOS for that episode of care was captured and no truncation was required.

Our main analyses excluded patients who had MHC Possible status (i.e., those patients who had an MHC diagnosis in the baseline period or in the study period, but not both). In another subsidiary analysis (see online Appendix), we repeated the main analysis in the initial cohort 
( $n=911,451$ ), calculating mean LOS as a function of MHC as a three-way variable (MHC Yes, MHC Possible, MHC No). Mean LOS for the MHC Possible group was consistently intermediate between that for the MHC Yes and MHC No groups. For example, for the MHC Possible group, mean LOS was 16.2 at step 1, stage A; 15.1 at step 1 , stage C; 90.3 at step 10 , stage A; and 34.4 at step 10 , stage $\mathrm{C}$.

\section{DISCUSSION}

Choices about what algorithm to use when identifying episodes of inpatient care substantially alter conclusions about the overall intensity of inpatient use and about MHC-related disparities in LOS. Not searching across all appropriate sources of data can lead to failure to capture a substantial amount of inpatient care, thus leading to underestimates of LOS. Decisions about how to process records can likewise influence calculated LOS. While other studies have documented that algorithm choice can influence conclusions drawn from VHA data [11-13], we are not aware of this result having been previously documented for LOS.

Researchers have access to many sources of data about VHA patients' nonambulatory care. Indeed, the large number of sources can bewilder investigators new to VHA administrative data, who may be unsure which files to select. Fortunately, the technical manuals developed by the Department of Veterans Affairs Information Resource Center (available at http://www.virec.research.va.gov/) and the Department of Veterans Affairs Health Economics Resource Center (available at http://www.herc.research.va.gov/) explain these files in detail. Our data provide further empiric information to help guide these decisions. First, our results confirm that adding more data sources identifies more inpatient days. Second, our results indicate that the EXT and Census files are especially important sources of incremental days of inpatient care. Third, our results indicate that adding more data sources also changes conclusions about the magnitude of effect (though not the direction of effect) of MHC on LOS. The step at which this has a particularly pronounced effect is the addition of EXT files, indicating that, compared with patients with no MHC, patients with MHC have disproportionately more frequent or prolonged stays in the long-term care setting.

Investigators using any VHA database need to examine data closely to determine whether data processing steps are necessary. In the case of inpatient files, our data indicate that in addition to the standard procedure of deleting pure duplicate records, investigators must account for overlapping stays (wherein a single day can be counted twice) and, for studies such as ours that focus on a single year of care, to truncate days falling before or after the FY of interest. Such pitfalls could, in some cases, reflect data quality problems, such as a data-entry error in admission or discharge date. However, in many cases, they may not represent deficits in the quality of VHA administrative data, but instead may reflect VHA clinical/administrative record-keeping practices. For example, a single stay could legitimately be recorded in more than one file if these files are used differently. Similarly, a fee basis stay (with the correct admission and discharge dates) could be filed in a subsequent year's records if a delay occurred in receipt of the bill from the outside vendor. Regardless of whether some of these factors represent data quality problems, investigators need to account for them; if not, some patients will have inflated estimates of LOS. Indeed, without such corrections, some patients will appear to be on inpatient status for more than 365 days in a single FY.

While the focus of this study is on the issue of algorithm choice for calculation of LOS, we use MHC-related disparities in LOS as a case study to illustrate what can happen if such issues are not considered. Health services researchers frequently examine disparities in processes and outcomes of care. Historically, interest in disparities related to characteristics like race, sex, and age has been great, but emerging evidence suggests that disparities related to MHC status are also common [9,24]. We demonstrated that the magnitude of MHC-related differences in LOS varied markedly as a function of LOS algorithm. Thus, the methodological issues raised here are not just theoretical: algorithm choice can have marked effects on conclusions in healthcare disparities research.

In the course of conducting analyses for this illustrative example, a subsidiary benefit was that informative findings about associations between MHC status and LOS emerged. Patients with MHC spent more of FY03 on inpatient status than did patients with no MHC; this was a consistent and robust finding across every algorithm examined. This finding is consistent with other studies that have shown heavier use of inpatient services by patients with MHC [6,14-19]. Our study also shows that some types of care (e.g., EXT) are associated with a disproportionately greater MHC effect. Another strength of our approach is that we distinguished between patients 
with stronger evidence of MHC (i.e., at least one MHC diagnosis at baseline in FY01-02 and at least one confirmatory MHC diagnosis in the study period, FY03) and patients with less certain (Possible) MHC status (i.e., presence of an MHC diagnosis either at baseline or in the study period, but not both). Our subsidiary analyses provide information about MHC Possible patients, a group that has not been well characterized in prior work. The MHC Possible group is likely heterogeneous and includes patients with an erroneous MHC diagnosis, with transient or resolved MHC, or with less severe MHC, as well as patients who receive part of their care outside the VHA system. Mean LOS for the MHC Possible group consistently fell between the mean LOS observed for the MHC Yes and the MHC No groups.

Interpretation of our findings is subject to several caveats. First, our aim was to calculate total number of days spent on inpatient status during FY03; values should not be interpreted as indicating total LOS across years. However, for 91 percent of records, the patient's complete stay was contained within FY03. Second, we did not use the VHA Decision Support System (DSS) Outpatient (OPAT) file as a data assembly step. In the OPAT file, Stay Type 42, Bedsection 80 refers to nursing home care reimbursed by VHA in any particular month. However, dates of admission and discharge could not be accurately generated from that source. Third, our focus was on VHA use. Depending on an investigator's study question, capturing inpatient days spent in other settings might also be important, such as days identified from Medicare claims data, which can be linked to VHA administrative data [25]. Fourth, because the purpose of our study was to identify periods during which the patient was on nonoutpatient status, our LOS calculations included both acute care and long-term care days. Studies focusing on one or the other setting might need to consider other methodological issues. For example, a patient's stay in a skilled nursing facility could have short gaps (e.g., for a brief acute care stay), which might not be captured with the databases used. Fifth, our main analyses excluded patients whose MHC status could not be ascertained with certainty (MHC Possible), so LOS estimates cannot be generalized to all VHA patients. Subsidiary analyses suggested that these excluded patients had intermediate LOS and that algorithm choice similarly affected LOS calculations for them. Sixth, MHC diagnoses came from ICD-9 diagnosis codes in VHA administrative data rather than from direct assessment of patients' MHC.
Given the known problem of underdiagnosis of MHC [26-27], some patients with MHC are likely included in the MHC No group. This would be expected to bias results toward the null.

This study examines methods that should be considered when an algorithm is developed that uses VHA data to calculate LOS. The specific algorithm selected will depend on the research question, such as-

- What types of inpatient care are of interest? For example, is the focus on acute care, extended care, care received on a fee basis outside of VHA or some combination of these sources? If rehabilitative/extended care is the focus, will additional sources (e.g., VHA EXT, fee basis, non-VHA and DSS OPAT files, as well as Medicare or Medicaid files) be queried, and how will multiyear stays be addressed?

- Is the focus on care received in a particular time interval (such as one FY) or on a full episode of inpatient care? If the former, will subsequent years' files be searched for stays recorded in a subsequent FY, and what is the expected incremental benefit versus cost of pulling data from multiple years? If the latter, how many years of data will be searched to identify the complete LOS, which could potentially span many years?

- Is the objective to characterize private sector inpatient care received as well, and if so, should other sources (such as Medicare claims data) be queried?

Careful consideration of these study design issues should yield an algorithm tailored to a particular study's objectives.

\section{CONCLUSIONS}

Accounting for the methodological issues raised here should help VHA health services researchers avoid pitfalls in calculation of VHA LOS, such as failure to capture care recorded in more obscure data sources (leading to underestimates of LOS) or duplicate counting of some days of care (leading to overestimates of LOS). This result is expected to support more robust estimates for economic analyses, since inpatient costs contribute disproportionately to total cost of VHA care. This result is also expected to enhance the accuracy of data VHA uses in its evidence-based efforts to redesign its healthcare delivery systems, which aim to improve the quality of care provided to veterans. 


\section{ACKNOWLEDGMENTS}

\section{Author Contributions:}

Study concept and design: S. M. Frayne, E. Berg, T. H. Holmes, R. Moos, D. R. Berlowitz, D. R. Miller, L. Pogach.

Acquisition of data: S. M. Frayne, E. Berg, D. R. Miller.

Analysis and interpretation of data: S. M. Frayne, E. Berg, T. H. Holmes, R. Moos, D. R. Berlowitz, D. R. Miller, L. Pogach, K. Laungani. Drafting of manuscript: S. M. Frayne, T. H. Holmes, E. Berg. Critical revision of manuscript for important intellectual content: S. M. Frayne, E. Berg, T. H. Holmes, R. Moos, D. R. Berlowitz, D. R. Miller, L. Pogach, K. Laungani, V. W. Jackson.

Statistical analysis: T. H. Holmes, E. Berg.

Obtained funding: S. M. Frayne.

Administrative, technical, or material support: E. Berg, K. Laungani. Study supervision: S. M. Frayne.

Financial Disclosures: The authors have indicated that no competing interests exist.

Funding/Support: This material was based on work supported in part by the National Institutes of Health (grant NIDDK 1 R01 DK07120201) and by VA Health Services Research and Development Service, (grant RCS 90-001).

Additional Contributions: The authors are grateful to Ciaran Phibbs, $\mathrm{PhD}$; Todd Wagner, $\mathrm{PhD}$; and Susan Schmitt, $\mathrm{PhD}$, for their advice and conceptual input during the process of developing the LOS algorithm. The views expressed in this article are those of the authors and do not necessarily represent the views of the VA.

\section{REFERENCES}

1. Moos RH, Mertens JR. Patterns of diagnoses, comorbidities, and treatment in late-middle-aged and older affective disorder patients: Comparison of mental health and medical sectors. J Am Geriatr Soc. 1996;44(6):682-88.

[PMID: 8642160]

2. Elixhauser A, Steiner C, Harris DR, Coffey RM. Comorbidity measures for use with administrative data. Med Care. 1998;36(1):8-27. [PMID: 9431328] DOI:10.1097/00005650-199801000-00004

3. Ronis DL, Bates EW, Garfein AJ, Buit BK, Falcon SP, Liberzon I. Longitudinal patterns of care for patients with posttraumatic stress disorder. J Trauma Stress. 1996;9(4): 763-81. [PMID: 8902745] DOI:10.1002/jts.2490090407

4. Saitz R, Ghali WA, Moskowitz MA. The impact of alcohol-related diagnoses on pneumonia outcomes. Arch Intern Med. 1997;157(13):1446-52. [PMID: 9224223] DOI:10.1001/archinte.157.13.1446

5. Cartwright WS, Ingster LM. A patient-based analysis of drug disorder diagnoses in the Medicare population. Health Care Financ Rev. 1993;15(2):89-101. [PMID: 10171899]
6. Ettner SL, Hermann RC. Inpatient psychiatric treatment of elderly Medicare beneficiaries. Psychiatr Serv. 1998;49(9): 1173-79. [PMID: 9735958$]$

7. Clague JE, Craddock E, Andrew G, Horan MA, Pendleton N. Predictors of outcome following hip fracture. Admission time predicts length of stay and in-hospital mortality. Injury. 2002;33(1):1-6. [PMID: 11879824] DOI:10.1016/S0020-1383(01)00142-5

8. Wigder HN, Johnson C, Shah MR. Length of stay predicts patient and family satisfaction with trauma center services. Am J Emerg Med. 2003;21(7):606-7. [PMID: 14655246$]$ DOI:10.1016/j.ajem.2003.08.019

9. Frayne SM, Halanych JH, Miller DR, Wang F, Lin H, Pogach L, Sharkansky EJ, Keane TM, Skinner KM, Rosen CS, Berlowitz DR. Disparities in diabetes care: Impact of mental illness. Arch Intern Med. 2005;165(22):2631-38.

[PMID: 16344421]

DOI:10.1001/archinte.165.22.2631

10. Hornbrook MC, Hurtado AV, Johnson RE. Health care episodes: Definition, measurement and use. Med Care Rev. 1985;42(2):163-218. [PMID: 10274864]

DOI:10.1177/107755878504200202

11. Frayne SM, Yano EM, Nguyen VQ, Yu W, Ananth L, Chiu VY, Phibbs CS. Gender disparities in Veterans Health Administration care: Importance of accounting for veteran status. Med Care. 2008;46(5):549-53. [PMID: 18438204] DOI:10.1097/MLR.0b013e3181608115

12. Halanych JH, Wang F, Miller DR, Pogach LM, Lin H, Berlowitz DR, Frayne SM. Racial/ethnic differences in diabetes care for older veterans: Accounting for dual health system use changes conclusions. Med Care. 2006;44(5):439-45.

[PMID: 16641662]

DOI:10.1097/01.mlr.0000207433.70159.23

13. Borzecki AM, Wong AT, Hickey EC, Ash AS, Berlowitz DR. Identifying hypertension-related comorbidities from administrative data: What's the optimal approach? Am J Med Qual. 2004;19(5):201-6. [PMID: 15532912]

DOI:10.1177/106286060401900504

14. Ashton CM, Petersen NJ, Wray NP, Yu HJ. The Veterans Affairs medical care system: Hospital and clinic utilization statistics for 1994. Med Care. 1998;36(6):793-803.

[PMID: 9630121$]$ DOI:10.1097/00005650-199806000-00003

15. Verbosky LA, Franco KN, Zrull JP. The relationship between depression and length of stay in the general hospital patient. J Clin Psychiatry. 1993;54(5):177-81. [PMID: 8509347]

16. Savoca E. Psychiatric co-morbidity and hospital utilization in the general medical sector. Psychol Med. 1999;29(2):457-64. [PMID: 10218937] DOI:10.1017/S0033291798008071 
17. Saravay SM, Steinberg MD, Weinschel B, Pollack S, Alovis N. Psychological comorbidity and length of stay in the general hospital. Am J Psychiatry. 1991;148(3):324-29. [PMID: 1992834]

18. Bressi SK, Marcus SC, Solomon PL. The impact of psychiatric comorbidity on general hospital length of stay. Psychiatr Q. 2006;77(3):203-9. [PMID: 16958003] DOI:10.1007/s11126-006-9007-X

19. Sayers SL, Hanrahan N, Kutney A, Clarke SP, Reis BF, Riegel B. Psychiatric comorbidity and greater hospitalization risk, longer length of stay, and higher hospitalization costs in older adults with heart failure. J Am Geriatr Soc. 2007;55(10):1585-91. [PMID: 17714458]

DOI:10.1111/j.1532-5415.2007.01368.x

20. Miller DR, Safford MM, Pogach LM. Who has diabetes? Best estimates of diabetes prevalence in the Department of Veterans Affairs based on computerized patient data. Diabetes Care. 2004;27 Suppl 2:B10-21. [PMID: 15113777$]$ DOI:10.2337/diacare.27.suppl_2.B10

21. Clinical Classifications Software (CCS) for ICD-9-CM [Internet]. Rockville (MD): Healthcare Cost and Utilization Project; 2008. Available from: http://www.hcup-us.ahrq.gov/toolssoftware/ccs/ccs.jsp.

22. Selim AJ, Fincke G, Ren XS. The comorbidity index. In: Goldfield N, Pine M, Pine J, editors. Measuring and managing health care quality: Procedures, techniques, and protocols. 2nd ed. New York (NY): Aspen; 2002.

23. Selim AJ, Fincke G, Ren XS, Lee A, Rogers WH, Miller DR, Skinner KM, Linzer M, Kazis LE. Comorbidity assessments based on patient report: Results from the Veterans Health Study. J Ambul Care Manage. 2004;27(3):281-95. [PMID: 15287217]

24. Druss BG, Bradford DW, Rosenheck RA, Radford MJ, Krumholz HM. Mental disorders and use of cardiovascular procedures after myocardial infarction. JAMA. 2000;283(4):
506-11. [PMID: 10659877]

DOI:10.1001/jama.283.4.506

25. Fleming C, Fisher ES, Chang CH, Bubolz TA, Malenka DJ. Studying outcomes and hospital utilization in the elderly. The advantages of a merged data base for Medicare and Veterans Affairs hospitals. Med Care. 1992;30(5):377-91. [PMID: 1583916] DOI:10.1097/00005650-199205000-00001

26. Kimerling R, Ouimette P, Prins A, Nisco P, Lawler C, Cronkite R, Moos RH. Brief report: Utility of a short screening scale for DSM-IV PTSD in primary care. J Gen Intern Med. 2005;21(1):65-67. [PMID: 16423126] DOI:10.1111/j.1525-1497.2005.00292.X

27. Pérez-Stable EJ, Miranda J, Muñoz RF, Ying YW. Depression in medical outpatients. Underrecognition and misdiagnosis. Arch Intern Med. 1990;150(5):1083-88.

[PMID: 2184790]

DOI:10.1001/archinte.150.5.1083

Submitted for publication August 3, 2009. Accepted in revised form January 13, 2010.

This article and any supplementary material should be cited as follows:

Frayne SM, Berg E, Holmes TH, Laungani K, Berlowitz DR, Miller DR, Pogach L, Jackson VW, Moos R. Mental illness-related disparities in length of stay: Algorithm choice influences results. J Rehabil Res Dev. 2010:47(8): 709-18.

DOI:10.1682/JRRD/2009.08.0112

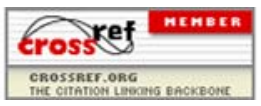

